\title{
Walk-Run Transition Speed Training as an Efficient Exercise Adjunct to Dietary Restriction in the Management of Obesity: A Prospective Intervention Pilot Study
}

\author{
Latifa Beltaifa Anis Chaouachi Rim Zérifi Lamia Boussaidi Ikram Bouzrati \\ Abdelmajid Abid Amina Elkhadi Karim Chamari Aly Raies \\ Tunesian Research Laboratory 'Sports Performance Optimisation', National Centre of Medicine and Science in Sports, Tunis, Tunisia
}

\section{Keywords}

Overweight · Gait speed - Weight loss · Health

\section{Summary}

Objective: The aim of this study was to test the utility of preferred walk-run transition speed (WRTS) in exercise training adjunct to dietary restriction for obesity management in healthy obese women. Materials and Methods: 37 obese women (age: $35 \pm 9$ years, body mass index (BMI): $34.9 \pm 4.6 \mathrm{~kg} / \mathrm{m}^{2}$ ) were assigned to an intervention pilot study during 6 months of restricted diet alone (RD) followed by 6 months of RD combined with WRTS (RD and WRTS) as a training exercise. Body mass, waist circumference (WC), fat mass (FM), fat free mass (FFM), active cell mass (ACM), fasting glucose, serum lipids (triacylglycerol (TG), total cholesterol (TC), high density lipoprotein cholesterol (HDL-C), apo-lipoproteins $A 1(A p o A 1)$ and $B(A p o B)]$, leptin and insulin concentrations, and HOMA-IR were assessed at baseline (TO), at the end of the RD alone (T1), and at the end of the RD and WRTS programme (T2). Results: Mean weight loss was $8.6 \pm 4.9 \mathrm{~kg}$ and $2.2 \pm 2.9 \mathrm{~kg}$ for (T0-T1) and (T1-T2), respectively. Significant BMI and WC reductions were reported at T1 and T2. FM decreased significantly both with RD and with RD and WRTS training whereas FFM and $A C M$ increased with RD and WRTS training only. TG decreased significantly with the two phases of the programme. A significant increase in HDL-C, and a decrease in LDL-C and TC/HDL-C ratio were noticed with $\mathrm{RD}$ and WRTS training. Heart rate monitored in training improved significantly after RD and WRTS training. A significant relationship ( $r=0.542, p<0.02$ ) was demonstrated between reductions in serum leptin and insulin concentrations observed with both RD and WRTS training. Conclusion: The addition of WRTS training to RD promoted a greater reduction in body mass, WC, FM, leptin and insulin concentrations, improved metabolic and cardiovascular risk factors, and enhanced cardiovascular fitness.

\section{Introduction}

Obesity has increased dramatically world-wide. It is one of the biggest health problems that affect developing and industrialized countries alike [1]. In Tunisia, a middle-income North African country, there is a greater prevalence of obesity in women compared with men [2]. Lifestyle factors, including high-energy diets and lack of physical activity, are major contributors to energy imbalance that causes overweight and obesity [1]. As a consequence, there is a world-wide rise in the reported incidence of diseases such as coronary heart disease, hypertension, diabetes, and some cancers that are associated with obesity [3, 4]. Therefore, there is an urgent need to change lifestyle and diet, and to increase physical activity for many of the world's population [1]. Different methods to lose weight have been proposed, but the most important factors are restricted energy diets and increased physical activity. Recent studies have suggested that it is not fat content but energy density that affects energy intake in obese women [5]. Some authors have proposed that reducing energy intake, by limiting food choice may lead to hunger that is associated with a lack of weight loss or even weight gain [6]. Another study proposed that food restriction is more effective than exercise

\section{KARGER}

Fax +497614520714

Information@Karger.de

www.karger.com (c) 2011 S. Karger GmbH, Freiburg

$1662-4025 / 11 / 0041-0045 \$ 38.00 / 0$

Accessible online at:

www.karger.com/ofa
Latifa Beltaifa

National Institute of Nutrion

11 rue Jebel Lakhdar, Bab Saadoun 1006, Tunis, Tunesia

Tel. +216-98216451, Fax -709789 


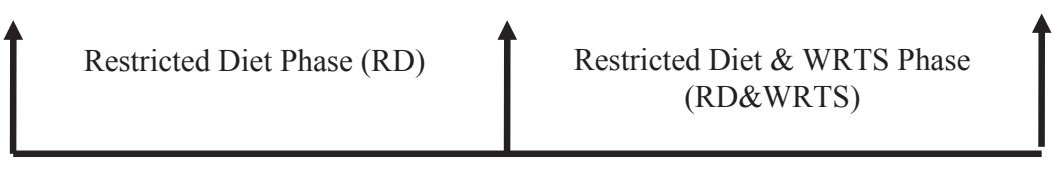

Fig. 1. Intervention study design.

T0: 0 months

T1: 6 months

T2: 12 months

in reducing body mass, while exercise is more effective in reducing body fat [7]. Shai et al. [8] suggested that personal preferences and metabolic needs might help in developing individualized tailoring of nutrition interventions. Physical activity is considered as an important tool in weight reduction and weight maintenance strategies [1]. Many exercise modes have been proposed including treadmill walking, cycle ergometer pedalling [9], and stepping [10]. Browning and Kram [11] suggested that walking may be an appropriate exercise recommendation for a weight management prescription in obese adults. The speed of transition between walking and running has been extensively investigated as a mode of exercise in several populations [11-13]. The majority of these studies have observed the walk-run transition speed (WRTS) as part of their research studies, but their main focus has been the measurement of energy expenditure [12] and/or the cost [11] of the cardio-respiratory responses $[9,13]$. To the best of our knowledge, no one has used WRTS as a mode of exercise in any long-term intervention studies. The aim of this pilot study was therefore to test the utility of using preferred WRTS in healthy, obese, dieting women as an adjunct in an obesity management programme. The data of this prospective study will offer a basis for the evaluation of the benefits of such a protocol.

\section{Participants and Methods}

Subjects

37 obese adult Tunisian sedentary women aged $20-50$ years with a body mass index $(\mathrm{BMI}) \geq 30 \mathrm{~kg} / \mathrm{m}^{2}$ volunteered to participate in the present obesity intervention pilot study. Obese women were recruited by advertising in a local newspaper. Exclusion criteria included pregnancy, breastfeeding, smokers, and anyone with obesity-associated pathology (e.g. diabetes, cardiovascular diseases, hypothyroidism, and psychiatric illness). All participants were classified at baseline as sedentary which was defined as reporting exercising less than 3 days/week for less than $20 \mathrm{~min} /$ day over the previous 6 months. Self reported physical activity was quantified by asking the subjects to recall their activities concurrently with each 3-day dietary record at T0 and T1 (data not shown). In addition, participants were eligible for inclusion if there was no evidence of their participation in diet reduction programmes within the last 6 months. All participants provided written informed consent before commencing the study. 37 participants began the study, 28 completed phase 1, and 26 finish the whole study (fig. 1). Participants completed a medical history and dietary questionnaires, and underwent anthropometric measurements and blood withdrawal for subsequent biochemical analysis. All outcome measures for the participants were assessed at baseline (T0), and at 6 months (T1), and 12 months (T2). The interven-
Table 1. Training programme

\begin{tabular}{lrrrr}
\hline & W1-2 & W3 & W4 & W5-24 \\
\hline Duration, min & 30 & 40 & 50 & 60 \\
Frequency, session/week & 3 & 3 & 3 & 3 \\
Intensity, \% WRTS & 100 & 100 & 100 & 100 \\
\hline
\end{tabular}

tion study consisted of an individualized restriction diet and an individualized WRTS training program.

\section{Individualized Diet Restriction Program (Phase 1)}

Food intake evaluation of each participant was determined at baseline (T0), after 6 month of restricted diet (RD) alone (T1), and at the end of the RD and WRTS intervention (T2) using 3-day diet records. Energy intake was compared to energy needs using the Schofield Equation for women [14]. Physical activity factor corresponding to sedentary state was used to calculate basal metabolic rate. A low energy diet was individually prescribed, and the restriction was $25-30 \%$ of the initial total energy intake. The RD used in the present study was a Mediterranean-style diet characterized by a relatively high carbohydrate (50-55\%), low fat ( $<30 \%$, especially monounsaturated fatty acids), and high fibre content with a low glycaemic index. The total energy intake (kcal/day) and the contributing percentage of the macronutrients fat, carbohydrates, and protein intake was calculated with the Bilnut 2.01 Software package (S.C.D.A. Nutrisoft, Cerelles, France) [15] using 230 added national Tunisian foods and meals [16]. To study the effects of RD alone over 6 months, subjects were counselled to maintain their habitual physical activity in order to eliminate the confounding effects of major weight loss or of body composition changes due to added physical activity/exercise during this period.

Association of WRTS Programme to RD (Phase 2)

In addition to the restricted diet programme described above, a physical activity intervention was included for a period of the remaining 6 months of the study. This was based on the individually preferred WRTS [11] defined as the lowest velocity at which the subjects chose to start running.

\section{Determination of WRTS}

10-min treadmill adaptation protocols were applied to all participants. Subsequently, WRTS was determined and its reproducibility verified on two occasions separated by a 48-hour interval. To determine WRTS, the following protocol was applied: a 10-min progressive warm up consisting of $4 \mathrm{~min}$ at $4 \mathrm{~km} / \mathrm{h}, 4 \mathrm{~min}$ at $5 \mathrm{~km} / \mathrm{h}$, and $2 \mathrm{~min}$ at $6 \mathrm{~km} / \mathrm{h}$. Participants then rested for $5 \mathrm{~min}$ to avoid a training effect. For the determination of the WRTS, the treadmill speed was initially set at a comfortable walking pace of $4.5 \mathrm{~km} / \mathrm{h}$. The protocol began at a speed of $4.5 \mathrm{~km} / \mathrm{h}$, the speed was increased incrementally by $0.2 \mathrm{~km} / \mathrm{h}$ every $30 \mathrm{~s}$ until the subject felt the need to run. Participants were asked to walk for as long as they could and to start running at the speed where they felt more comfortable running. At this point, the subject was asked to run for $30 \mathrm{~s}$ and then to walk for $30 \mathrm{~s}$ at the same speed. The speed was then reduced to $4 \mathrm{~km} / \mathrm{h}$ for $2 \mathrm{~min}$ before stopping the treadmill. These walk-run intervals were repeated at 
Table 2. Characteristics of subjects at baseline (T0) after $\mathrm{RD}$ alone (T1) and after RD and WRTS programme (T2) for the 26 subjects who completed the entire study programme (data are means $\pm \mathrm{SD}$ )

\begin{tabular}{llllll}
\hline & $\begin{array}{l}\mathrm{T} 0 \\
(\mathrm{n}=26)\end{array}$ & $\begin{array}{l}\mathrm{T} 1 \\
(\mathrm{n}=26)\end{array}$ & $\mathrm{p}$ value & $\begin{array}{l}\mathrm{T} 2 \\
(\mathrm{n}=26)\end{array}$ & p value \\
\hline Body mass, kg & $91.7 \pm 14.4$ & $80.4 \pm 11.6$ & 0.000 & $78.6 \pm 10.8$ & 0.035 \\
BMI, kg/m ${ }^{2}$ & $34.9 \pm 4.6$ & $31.2 \pm 4.2$ & 0.000 & $30.5 \pm 3.7$ & 0.033 \\
WC, cm & $105.5 \pm 10.8$ & $96.2 \pm 8.9$ & 0.000 & $91.2 \pm 10.6$ & 0.002 \\
Fat mass, \% & $48.2 \pm 6.7$ & $41.9 \pm 5.1$ & 0.000 & $37.2 \pm 7.2$ & 0.000 \\
Fat free mass, \% & $46.1 \pm 5.0$ & $40.8 \pm 3.7$ & 0.000 & $43.0 \pm 4.5$ & 0.01 \\
Active cell mass, kg & $31.2 \pm 4.6$ & $30.6 \pm 4.5$ & $\mathrm{NS}$ & $32.2 \pm 6.5$ & 0.021 \\
Fasting glucose, mmol/1 & $5.8 \pm 0.7$ & $5.7 \pm 0.7$ & $\mathrm{NS}$ & $5.4 \pm 0.6$ & 0.001 \\
Triacylglycerol, mmol/l & $1.31 \pm 0.4$ & $1.11 \pm 0.4$ & 0.000 & $0.85 \pm 0.3$ & 0.001 \\
Total cholesterol, mmol/1 & $4.7 \pm 0.8$ & $4.5 \pm 0.7$ & $\mathrm{NS}$ & $4.4 \pm 0.6$ & 0.011 \\
HDL-C, mmol/l & $1.37 \pm 0.2$ & $1.27 \pm 0.2$ & $\mathrm{NS}$ & $1.64 \pm 0.2$ & 0.000 \\
LDL-C, mmol/l & $2.71 \pm 0.7$ & $2.78 \pm 0.6$ & $\mathrm{NS}$ & $2.42 \pm 0.6$ & 0.001 \\
Apo-lipoprotein A1 & $1.7 \pm 0.3$ & $1.69 \pm 0.24$ & $\mathrm{NS}$ & $1.67 \pm 0.35$ & $\mathrm{NS}$ \\
Apo-lipoprotein B & $0.66 \pm 0.02$ & $0.65 \pm 0.01$ & $\mathrm{NS}$ & $0.73 \pm 0.03$ & $\mathrm{NS}$ \\
TC/HDL-C ratio & $3.75 \pm 0.7$ & $3.65 \pm 0.5$ & $\mathrm{NS}$ & $2.74 \pm 0.4$ & 0.001 \\
LDL-C/HDL-C ratio & $2.07 \pm 0.7$ & $2.26 \pm 0.6$ & $\mathrm{NS}$ & $1.50 \pm 0.4$ & 0.000 \\
HOMA-IR & $4.5 \pm 1.4$ & $3.7 \pm 0.9$ & 0.001 & $2.9 \pm 0.7$ & 0.000 \\
Leptin, ng/ml & $39.5 \pm 14.5$ & $27.7 \pm 10.3$ & 0.012 & $23.5 \pm 9.8$ & 0.000 \\
Insulin, $\mu$ UU/ml & $17.5 \pm 4.8$ & $14.2 \pm 3.7$ & 0.000 & $11.6 \pm 2.9$ & 0.006 \\
RHR, bpm & - & $88.8 \pm 11$ & & $82.3 \pm 11.7$ & 0.005 \\
HR at walking, bpm & - & $146.9 \pm 16$ & & $138.2 \pm 13$ & 0.000 \\
HRpeak, bpm & - & $165.7 \pm 10.1$ & & $157.6 \pm 13.2$ & 0.003 \\
WRTS, km/h & - & $6.98 \pm 0.4$ & & $8.09 \pm 0.6$ & 0.000 \\
\hline
\end{tabular}

HDL-C = high-density lipoprotein cholesterol; HOMA-IR = homeostasis model assessment index for insulin resistance; LDL-C = low-density lipoprotein cholesterol; NS = not significant; RHR = resting heart rate; HR = heart rate, HRpeak $=$ heart rate peak; $\mathrm{WC}=$ waist circumference .

least twice until the subject was sure of the particular speed where running was preferred. If they decided that walking was preferred, the treadmill speed was increased to the next higher level, and the walk-run $30 \mathrm{~s}$ interval procedure was repeated until the subject was certain when running was the preferred mode. The preferred WRTS was calculated as the mean of the 2 or 3 trials separated by a 5 -min rest to avoid a training effect. The speed at which the individual transitioned from a walk to a run and continued running for the main part was defined as the individual's WRTS. At 3-month intervals during the RD and WRTS training, tests to adjust WRTS were performed. The training consisted of continued walking sessions with controlled speed at $100 \%$ of WRTS. The exercise was carried out 3 times a week, and the duration of each session was gradually increased from approximately $30 \mathrm{~min}$ to $60 \mathrm{~min}$ by the end of the study (table 1). Exercise duration was determined following the recommendations for adults from the ACSM/AHA [17]. Because of their initial sedentary status, participants were assigned to a progressive weight training programme. Each exercise session was supervised by the primary investigator and two other associate investigators. Tests to determine the WRTS were performed on a treadmill, and all the training sessions took place on an overground 100-metre walkway at the gymnasium of the National Centre of Medicine and Science in Sports of Tunis to eliminate wind resistance or inclement weather. Heart rate (HR) was monitored during WRTS tests and during each training session using a cardio-frequency meter (Polar S 810TM, Kemple, Finland). The HR peak (HRpeak) was taken as the highest HR achieved during the training. Resting HR (RHR) was recorded before the tests and before the training sessions. HR readings were directly downloaded to a computer via a serial interface.

\section{Anthropometrical Measurements}

Body mass was measured with the subjects barefoot and wearing very light clothing, to an accuracy of $0.1 \mathrm{~kg}$ using foot-to-foot bio-electrical impedance analysis (Model TBF 300, Tanita Inc., Tokyo, Japan). Height was recorded to the nearest $0.5 \mathrm{~cm}$ barefoot using a fixed stadiometer (Seca, Model 220, Hamburg, Germany). Waist circumference (WC) was measured with a non-expandable tape at the point of the umbilicus, to the nearest millimetre with the subject in the standing position using the WC Tunisian cut-off protocol of the National Nutrition Survey 1996/97 [18]. Body composition was measured using the bioelectrical impedance analysis system (BIA) (Analycor 4, Spengler, Cachan, France) and performed according to the manufacturer's instructions using a multifrequency of 5, 50 , and $100 \mathrm{kHz}$. The day before the impedance measurements, subjects were instructed to drink sufficient fluids to be well hydrated. The procedure was carried out after the patient had been resting in a supine position for at least $30 \mathrm{~min}$. Percentages of fat mass (FM), fat free mass (FFM), and active cell mass (ACM) were determined. ACM was defined as the difference between FFM and the sum of extracellular fluid and solids.

\section{Biochemical Analysis}

Serum and plasma samples (obtained by centrifugation at $4{ }^{\circ} \mathrm{C}$ ) were taken after an overnight fast at $\mathrm{T} 0, \mathrm{~T} 1$, and $\mathrm{T} 2$ and stored frozen at minus $80^{\circ} \mathrm{C}$ until assayed. Serum glucose concentration, serum total cholesterol (TC), high-density cholesterol (HDL-C), triacylglycerol (TG), thyroidstimulating hormone, serum insulin, and serum leptin concentrations were analysed at the biochemical laboratory of the National Institute of Nutrition, Tunis. Serum glucose concentration was measured by enzymatic colorimetric methods (glucose oxidase), serum TC (cholesterol oxidase) and concentrations of serum total TG (glycerol kinase) using a Beckman CX9 analyser (Beckman Coulter, Inc. Brea, CA, USA). HDL-C was measured enzymatically (using direct method); concentrations of low-density cholesterol (LDL-C) were calculated using Friedewald's validated equation [19]: LDL-C $(\mathrm{mmol} / \mathrm{l})=\mathrm{TC}-(\mathrm{TG} / 2.2+\mathrm{HDL}-$ C). Serum insulin levels were determined using an immunochemiluminescence assay (DPC Immulite 1000 Immunoassay Analyser, Siemens Medi- 
Fig. 2. Percentage changes in lipid profile and insulin sensitivity between $\mathrm{T} 1$ and $\mathrm{T} 0$ (month 6 and month 0 ) and between $\mathrm{T} 2$ and $\mathrm{T} 1$ (month 12 and month 6). RD versus RD and WRTS. $\mathrm{TG}=$ Delta triacylglycerol; $\mathrm{TC}=$ delta total cholesterol, HDL = delta high-density lipoprotein cholesterol; LDL = delta low-density lipoprotein cholesterol; TC/HDL $=$ TC/HDL ratio; HOMA-IR = delta homeostasis model assessment index for insulin resistance.

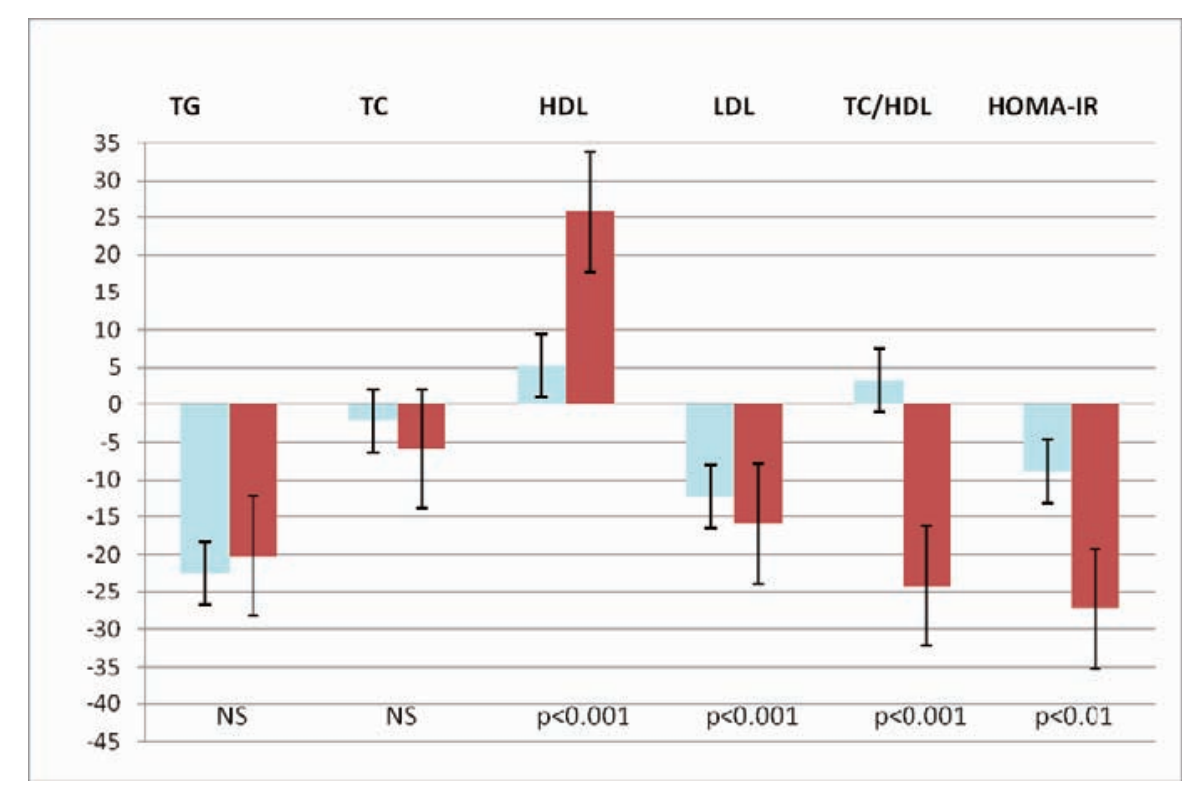

cal Solutions Malvern, PA, USA). The normal range for insulin levels was 2-25 $\mu \mathrm{U} / \mathrm{ml}$. Insulin resistance (IR) was estimated by the homeostasis model assessment (HOMA) index and calculated using the formula: HOMA-IR $=$ insulin $(\mathrm{mU} / \mathrm{l}) \times$ fasting glucose $(\mathrm{mmol} / \mathrm{l}) / 22.5$ [20]. Serum leptin concentrations were measured using radioimmunoassay (Human Leptin RIA Kit, Linco Reaserch, St. Charles, MO, USA). The values measured were in the range of detection (range $0.5-100 \mathrm{ng} / \mathrm{ml}$ ). All determinations of leptin levels were run in a single assay to eliminate interassay variation. All the measurements of the study were performed by the same experienced investigator.

\section{Statistical Analysis}

Statistical analysis was carried out using the SPSS/PC Statistical Package for Social Science version 13.0 (SPSS Inc., Chicago, IL, USA). Data were presented as means \pm standard deviation (SD). Friedman's two-way analysis of variance (ANOVA) for repeated measures was used to determine the differences between the phases of the study, with each subject serving as his own control. When a significant $\chi^{2}$ value was achieved, appropriate post-hoc test procedures were used to locate the difference between baseline and 6-month obesity intervention study and between the 6-month and 12-month intervention study. Pearson correlation was used to study the relations between changes variables over time. Test-retest reliability of the WRTS test was determined by intra-class correlation coefficients (ICC) via one-way ANOVA with a value of $0.7-0.8$ being questionable, and $>0.9$ indicating high reliability [21]. The significance threshold was set at $\mathrm{p}<0.05$.

\section{Results}

37 participants completed the measurements at baseline; only 28 completed the RD alone. Nine participants left the RD and WRTS phase (fig. 1). There were no statistically significant differences with respect to age, body mass, BMI, and body composition between the group of 28 subjects who completed the second assessment (RD and WRTS) and the 9 subjects who did not. Of the 28 subjects who entered the RD and WRTS phase, only 26 completed the whole phase and did all the exercise training. At baseline, characteristics of the par-

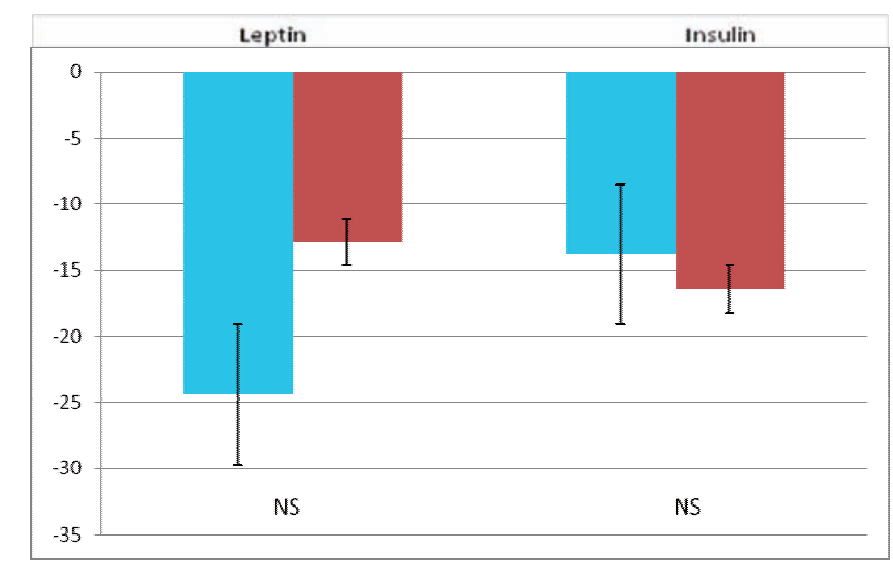

Fig. 3. Percentage variations in serum leptin and serum insulin concentrations between T1 and T0 (month 6 and month 0 ) and between T2 and $\mathrm{T} 1$ (month 12 and month 6). RD versus RD and WRTS.

ticipants are shown in table 2. Compared with the requirement needs, there were no significant differences between the mean energy deficit $(757 \pm 393 \mathrm{kcal} /$ day $)$ for women younger than 30 years and for women aged 30 years or more $(657 \pm 315 \mathrm{kcal} / \mathrm{day})$. The corresponding fat intakes were 25.8 $\pm 3.9 \%$ and $23.1 \pm 2.6 \%$, and for carbohydrates $53.2 \pm 7.6 \%$ and $50.2 \pm 5.3 \%$ of the daily total energy intake, respectively. After the training programme, mean WRTS was faster $(\mathrm{p}<0.0001)$ than before the training $(1.92 \pm 0.07 \mathrm{~m} / \mathrm{s}$ vs. $1.44 \pm$ $0.02 \mathrm{~m} / \mathrm{s})$. The ICC values for the preferred WRTS measurement protocol demonstrated high reliability $(\mathrm{ICC}=0.97)$. Attendance for the exercise sessions averaged $88 \%$.

\section{Effect of Restricted Diet Alone}

Changes associated with this part of the programme are summarised in table 2. After the 6-month RD phase, body mass showed a significant reduction $(-8.8 \pm 5.3 \%)$. Overall, $18.5 \%$ 
of participants decreased their weight by more than $5 \%$ of their initial body mass, whereas $23 \%$ showed a greater than $10 \%$ weight loss. Only TG concentrations decreased significantly after RD alone compared with the baseline levels $(-22.5 \pm 8.6 \% ; p<0.005)$ (fig. 2). There was a significant reduction in serum leptin and serum insulin concentrations $(-24.4 \pm 10.8 \% ;-14.8 \pm 8.5 \%$ respectively) (fig. 3$)$, and a decrease in HOMA-IR $(4.5 \pm 1.4 \%$ vs. $3.7 \pm 1.0 \% ; \mathrm{p}<0.0001)$ following RD alone (fig. 2).

\section{Effect of WRTS Associated with Restricted Diet}

In the RD and WRTS phase of the study, 35\% of the participants lost less than $5 \%$ body mass, and $8.5 \%$ lost $5-10 \%$ of body mass. Significant differences were shown for reductions in body mass $(\mathrm{p}<0.035)$, BMI $(\mathrm{p}<0.033)$, WC $(\mathrm{p}<0.002)$, and body fat $(\mathrm{p}<0.0001)$ after RD and WRTS (table1). While FM decreased during this phase $(p<0.0001)$, FFM $(\mathrm{p}<0.0001)$ and ACM $(\mathrm{p}<0.021)$ increased significantly (table 1). Walking training associated with RD showed beneficial effects on HR: obese women significantly $(p<0.004)$ decreased their mean RHR as well as their mean exercise HR $(\mathrm{p}<0.01)$.

\section{Blood Analysis}

Fasting glucose decreased significantly during both the RD alone $(-1.2 \pm 2.6 \%)$ and RD and WRTS $(-8.8 \pm 8.7 \%)$ phases with the greater reduction occurring over the second 6 months $(\mathrm{p}<0.0001)$. The results in the HOMA-IR followed a similar pattern $(-8.9 \pm 6.7$ vs. $-27.2 \pm 12.8 \%, \mathrm{p}<0.01)$. The RD and WRTS programme significantly reduced serum TG concentrations $(-20.2 \pm 12.2 \%)$ while promoting an increase in HDL-C (+25.8 $\pm 14.8 \%)$ (fig. 2). Serum leptin and insulin concentrations decreased after RD alone $(\mathrm{p}<0.012$, $\mathrm{p}<0.001)$ and after RD and WRTS ( $p<0.0001, \mathrm{p}<0.006)$ (fig. 3). A significant relationship $(\mathrm{r}=0.542, \mathrm{p}<0.02)$ was demonstrated between reductions in serum leptin and insulin concentrations in response to RD and WRTS training.

\section{Discussion}

The present study reports data from a prospective intervention study investigating the effects of WRTS in conjunction with RD on weight loss, body composition, lipid profile, leptin and insulin concentrations in an obese population. To the best of our knowledge, this is the first study designed to test the efficacy and utility of using WRTS as an exercise training modality combined with reduced energy diets for obesity management. The important finding of this study is when WRTS training was carried out in conjunction with $\mathrm{RD}$, the results demonstrated improvements in body composition, and bigger reductions in lipid profiles as well as leptin and insulin concentrations. The second finding was that significant reductions in mean resting and exercise heart rates occurred, suggesting that obese women have the ability to induce significant improvements in physical and cardiovascular fitness after RD associated with WRTS training.

The current study also developed a reproducible protocol for WRTS treadmill determination in obese subjects. There is little research on the reproducibility of WRTS, although recently Farinatti and Monteiro [13] made a proposal for young and older subjects. There was no difference between mean WRTS measured in our obese participants $(1.44 \mathrm{~m} / \mathrm{s})$, and those of Browning and Kram [11] (1.40 m/s). However, Granley et al. [22] showed a higher mean WRTS with normal weight subjects $(2.08 \mathrm{~m} / \mathrm{s})$. When WRTS was expressed in $\mathrm{km} / \mathrm{h}$, Faranitti and Monteiro [13] found no difference between the old (6.84) and young groups (7.04), and these WRTS are not different from those of the present study (6.98 $\mathrm{km} / \mathrm{h}$ ). It has been shown that obese women prefer walking slowly [11], however, the present study has demonstrated that after a 3 sessions/week WRTS training programme during 6 months, WRTS improved and is a feasible exercise intensity for obesity management. While scientific literature [23, 24] has reported considerable functional limitations in motor activity in obese subjects, WRTS training combined with a restricted diet for 6 months in the present study improved the mean WRTS itself, suggesting that obese participants could attain higher levels of WRTS and improve their capacity for physical effort, which could then positively affect their quality of life and develop working ability.

The present study demonstrates that the addition of WRTS as exercise training, in conjunction with a $25-30 \%$ daily energy deficit was effective in significantly decreasing $\mathrm{WC}$ and FM, while increasing FFM and ACM (+4.66\%). However, weight loss was modest in this phase $(-2.2 \pm 2.9 \%)$ compared with $\mathrm{RD}$ alone $(-8.8 \pm 4.2 \% ; \mathrm{p}<0.005)$. It has been previously reported that programmes including simultaneous diet restrictions and exercise produce greater weight loss than diet alone in obese and overweight persons [25, 26]. However, some clinical studies have reported a compensatory relationship, such that obese and sedentary individuals increased their energy intake to compensate for the increase in exercise [27]. Women have been shown to compensate for increased energy expenditure by increasing their energy intake [27]. To avoid this interaction effect, the present study chose to investigate the effects of diet alone by the addition of exercise to the same restricted dietary intervention in the same group of obese women. Diet appears to be the most powerful component of weight loss; however, the additional role of exercise provides some evidence for an increased fat loss and continued metabolic improvement. Our results are consistent with the literature $[28,29]$ supporting reduced body fat, especially central body fat, as an independent risk factor for cardiovascular disease and suggesting that exercise is inclined to be optional with diet therapy to reduce and maintain WC and FM reductions. Potential interaction effects of diet and exercise on weight loss should be investigated, and a state of equilibrium 
must generally be expected after 6 months on an energy restricted diet. Different studies have examined if the effects of the two methods (diet and physical activity) were comparative or cumulative. It has been suggested that for women, the cumulative effect of concomitant changes in diet and exercise on weight loss are more than additive [26]; while other studies suggested that the relationship may be synergic [30], or compensatory [27]. In our prospective study, the correlations between some variables (e.g. insulin and leptin) during the two phases of the intervention programme lead us to speculate that there may be additional, interactive, and cumulative relationships between the effect of dietary and exercise change such that WRTS was required to enhance the effect of energy restriction, while RD enhanced the effects of WRTS by at least weight/fat loss.

Another relevant finding of the current study was the significant improvement in HR during exercise. It has been reported by different groups that the mean HR is often high in obese people and that obese women have significantly higher HR than their normal weight counterparts under the same exercise conditions [9]. During walking, HR responses were reported to be higher in an obese population compared with lean subjects [23]. Obesity is characterised by a decline in parasympathetic power accompanied by a rise in mean HR. This system participates in the control of glucose and fat metabolism, and lower levels of sympathetic activity are associated with lower energy expenditure and therefore a positive energy balance and potential weight gain [31]. Dominance of the sympathetic nervous system (SNS) over the parasympathetic nervous system (PNS) has been shown to be a strong risk factor for cardiovascular disease [32]. Obesity is associated with increased SNS activity, and weight loss seems to have positive effects on this balance. It is also known that among the beneficial impacts of weight loss on the cardiovascular system is a decrease in both RHR and HR variability [33]. One study reported an improvement in HR recovery following an energy restricted weight loss programme, due mainly to improvements in metabolic parameters (body mass, BMI, WC, TG, glucose, and TG/HDL-C ratio) [34]. In contrast, another study reported improvements in HR recovery after an exercise weight loss programme without any change in energy intake [35]. This resulted from a decrease in RHR regardless of weight loss and improvement in cardiovascular fitness [35]. Nevertheless, it seems that weight loss improves the SNS/PNS balance especially when energy restriction is combined with exercise $[31,32]$. It is widely assumed that good physical fitness and regular exercise training such as that carried out by obese subjects during 6 months in the present study induce adaptation of the autonomic nervous system, which is most commonly observed in the form of a decrease in RHR. This is mediated in part by an increase in cardiac vagal tone, which is mainly due to improvements in cardiopulmonary function promoted by exercise rather than amelioration of metabolic factors, including weight loss [31].
Leptin has been suggested to play a role in regulating body fat content, energy balance, and food intake [36]. Human obesity is characterised by high levels of circulating leptin, and obese females generally show higher plasma leptin concentrations in comparison with obese males [37]. Because of the low availability of glucose, insulin concentration decreases during periods of energy restriction [38]. Intervention studies using restriction diet and exercise have produced conflicting results. In the present study, both RD and RD plus WRTS training decreased serum leptin concentrations, while the combination of exercise training with dietary restriction promoted a greater decrease in serum glucose and insulin than did RD alone. These results are in accordance with current literature $[8,38,39]$. Because variations in circulating leptin concentrations are correlated with changes in insulin levels during weight loss, it may mean that leptin decreases the secretion of insulin by reducing the action of insulin on adipocytes [40]. It was also shown in an oxidative muscle type study that leptin can reduce intramuscular triglyceride stocks by increasing their hydrolysis and increasing fatty acid oxidation [41]. Thus, there appears to be a relationship between the reduction in leptin and that of insulin mediated by diet and exercise. In rodents, leptin acts via hypothalamic receptors to inhibit feeding and increase thermogenesis. A feedback regulatory loop whereby leptin production by adipose cells monitors the size of the adipose tissue, then through leptin receptors in the hypothalamus the leptin signal is integrated and operates effector mechanisms, including the SNS, controls the determinants of energy balance [42]. While this mechanism is not so clearly defined in humans [42], low energy diet induced weight loss has been associated with decreases in circulating leptin concentrations, reductions in whole body and muscle sympathetic nerve activity, and enhanced insulin sensitivity [43]. The decreases in leptin, insulin, and glucose levels with the concomitant reduction in RHR and HR during exercise in the present study, could therefore be interpreted as an effect of a reduction in the leptin signal decreasing sympathetic nerve activity and hence HR [44]. The results of the present study also show that RD and WRTS training consecutive to RD alone improved HOMA-IR in our obese participants, which might indicate an improvement in insulin sensitivity over the last 6 months of this investigation.

Obesity is considered as a major independent risk factor for cardiovascular diseases $[1,4]$. Studies have demonstrated that generally an increase in physical activity combined with $\mathrm{RD}$ improves the lipid profile compared with RD alone or physical activity alone [45, 46]. Although our participants did not present any metabolic related disorders at baseline, we observed significant improvements in lipid profiles. Both RD alone and consecutive RD and WRTS training had similar effects in reducing serum TG concentrations and lowering circulating LDL-C levels, but only the combination of exercise training with energy restriction promoted increases in HDL-C. An explanation must lie in both dietary intervention 
and training exercise. Dietary intervention associated with weight loss reduces mainly the mass of TG-rich very-low-density lipoprotein (VLDL) particles as well as the mass of LDL and large buoyant LDL particles [47]. The only potential mechanism through which exercise could affect LDL-C and HDL-C metabolism is by promoting fat utilization as a substrate and thereby creating a physiologic state conductive to enhance lipid metabolism [45, 46, 48]. It is well documented that gait transition from walking to running increases the rate of energy expenditure (EE) [23, 49]. Metabolic rate during walking speeds was $11 \%$ greater for the obese subjects compared with that of normal weight subjects [11]. A recent study revealed that essentially all of the extra energy required for transition from walking to running was provided by lipid oxidation [22]. In addition to metabolic factors, biomechanical components could also influence EE in obese subjects when walking at different speeds. Thus, increased recruitment of muscle fibre, increased power output, and mediolateral forces have been postulated as variables affecting locomotor economy that can increase EE during exercise [50].

One limitation of the present study is the relatively small study population at the end of the protocol. This was in spite of only $10 \%$ of participants dropping out during the RD only phase and $2 \%$ dropping out during the RD and WRTS phase for different reasons (lack of time, transport difficulties, pregnancy, but also pain related to WRTS training $(\mathrm{n}=2))$. However, this is acknowledged as a common characteristic of all longitudinal studies [25]. In the present study, we can suggest that the rate of initial weight loss during the 6 months of RD alone played an important role in the completion or not of the whole programme and acted as a confounding factor. Those who had a significant weight loss may have left the study as they had met their goal, while those who did not lose enough weight may have left because they were dissatisfied with their progress. Thus, it is important to undertake corrective measures to increase the likelihood of completion in such longterm studies.

In summary, the present study was designed to determine the efficacy and utility of a personalized weight management protocol using WRTS associated with RD. The addition of individualized WRTS exercise to a restricted diet induced additional weight/fat loss that was successful in improving the lipid profile, insulin sensitivity, and HR responses. Our findings suggest also that adding WRTS to RD exercise can produce substantial improvements in an individual's capacity for physical effort, and can improve several metabolic and cardiovascular risk factors in obese sedentary women.

\section{Acknowledgement}

This study was supported by the National Institute of Nutrition of Tunisia and the Tunisian Ministry of Scientific Research, Technology and Development of Competences. The authors would like to thank all the women who participated in this study. We especially thank ASSISLAB for providing Leptin Kits; Prof. Bechir Zouari for his assistance with statistics; Prof. M Chiheb Ben Rayana for his vital role in the chemical assays, and Dr. Zakia Bartagi for her valuable support before and during the experiment. Finally, the authors would like to thank Dr. John Leiper for revising the English and for his assistance during the revision of this manuscript.

\section{Disclosure Statement}

The authors declare no conflict of interest.

\section{References}

1 WHO: Obesity: preventing and managing the global epidemic: report of a WHO consultation. World Health Organ Tech Rep Ser 2000;894(i-xii): $1-253$.

2 Beltaifa L, Traissac P, El Ati J, Lefevre P, Romdhane H B, Delpeuch F: Prevalence of obesity and associated socioeconomic factors among Tunisian women from different living environments. Obes Rev 2009;10:145-153.

$\checkmark 3$ Larsson SC, Wolk A: Obesity and colon and rectal cancer risk: a meta-analysis of prospective studies. Am J Clin Nutr 2007;86:556-565.

4 Reisin E, Jack AV: Obesity and hypertension: mechanisms, cardio-renal consequences and therapeutic approaches. Med Clin North Am 2009;93:733-751.

$\checkmark 5$ Rolls BJ, Bell EA, Castellanos VH, Chow M, Pelkman CL, Thorwart ML: Energy density but not fat content of foods affected energy intake in lean and obese women. Am J Clin Nutr 1999;69:863-871.

6 Elfhag K, Rossner S: Who succeeds in maintaining weight loss? A conceptual review of factors associated with weight loss maintenance and weight regain. Obes Rev 2005;6:67-85.
7 Tsai AC, Sandretto A, Chung YC: Dieting is more effective in reducing weight but exercise is more effective in reducing fat during the early phase of a weight-reducing programme in healthy humans. J Nutr Biochem 2003;14:541-549.

8 Shai I, Schwarzfuchs D, Henkin Y, Shahar DR, Witkow S, Greenberg I, Golan R, Fraser D, Bolotin A, Vardi H, Tangi-Rozental O, Zuk-Ramot R, Sarusi B, Brickner D, Schwartz Z, Sheiner E, Marko R, Katorza E, Thiery J, Fiedler GM, Blüher M, Stumvoll M, Stampfer MJ: Weight loss with a low-carbohydrate, Mediterranean, or low-fat diet. N Engl J Med 2008;359:229-241.

9 Lafortuna CL, Agosti F, Galli R, Busti C, Lazzer $\mathrm{S}$, Sartorio A: The energetic and cardiovascular response to treadmill walking and cycle ergometer exercise in obese women. Eur J Appl Physiol 2008; 103:707-717.

10 Chen KY, Acra SA, Donahue CL, Sun M, Buchowski MS: Efficiency of walking and stepping: relationship to body fatness. Obes Res 2004;12: 982-989.

11 Browning RC, Kram R: Energetic cost and preferred speed of walking in obese vs. normal weight women. Obes Res 2005;13:891-899.
12 LeCheminant JD, Heden T, Smith J, Covington NK: Comparison of energy expenditure, economy, and pedometer counts between normal weight and overweight or obese women during a walking and jogging activity. Eur J Appl Physiol 2009;106:675-682.

13 Farinatti PT, Monteiro WD: Walk-run transition in young and older adults: with special reference to the cardio-respiratory responses. Eur J Appl Physiol 2010;109:379-388.

14 James WPT, Schofield E: Different levels of analysis in estimating requirements; in James WPT, Schofield E (eds): Human Energy Requirements, 1st ed. New York, NY, Oxoford Medical Publications, 1990, pp. 42-57.

15 Bourgette P, Roshaussen M: Nutrisoft-Bilnut: programme d'enquêtes alimentaires. Version 2.01. 1990.

16 Beltaifa L: Nos mets à la calorie prés, 1st ed. Tunis, Tunisia, Simpact Press, 2007.

17 Haskell WL, Lee IM, Pate RR, Powell KE, Blair SN, Franklin BA, Macera CA, Heath GW, Thompson PD, Bauman A: Physical activity and public health: updated recommendation for adults from the American College of Sports Medicine and the American Heart Association. Med Sci Sports Exerc 2007;39:1423-1434. 
18 Bouguerra R, Alberti H, Smida H, Salem L B, Rayana C B, El Atti J, Achour A, Gaigi S, Slama $\mathrm{CB}$, Zouari B, Alberti KG: Waist circumference cut-off points for identification of abdominal obesity among the Tunisian adult population. Diabetes Obes Metab 2007;9:859-868.

19 Friedewald WT, Levy RI, Fredrickson DS: Estimation of the concentration of low-density lipoprotein cholesterol in plasma, without use of the preparative ultracentrifuge. Clin Chem 1972;18:499-502.

20 Haffner SM, Miettinen H, Stem MP: The Homeostasis model in the San Antonio Heart Study. Diabetes Care 1997;20:1087-1092.

21 Vincent W: Statistics in Kinesiology, 3rd ed. Champaign, IL, Human Kinetics, 2005.

22 Ganley KJ, Stock A, Herman RM, Santello M, Willis WT: Fuel oxidation at the walk-to-run-transition in humans. Metabolism 2010; Epub ahead of print.

23 Greiwe JS, Kohrt WM: Energy expenditure during walking and jogging. J Sports Med Phys Fitness 2000;40:297-302.

-24 Lafortuna CL, Fumagalli E, Vangeli V, Sartorio A: Lower limb alactic anaerobic power output assessed with different techniques in morbid obesity. J Endocrinol Invest 2002;25:134-141.

25 Curioni CC, Lourenço PM: Long-term weight loss after diet and exercise: a systematic review. Int J Obes (Lond) 2005;29:1168-1174.

26 Dunn CL, Hannan PJ, Jeffery RW, Sherwood NE, Pronk NP, Boyle R: The comparative and cumulative effects of a dietary restriction and exercise on weight loss. Int J Obes 2006;30:112-121.

27 Woo R, Garrow JS, Pi-Sunyer FX: Effect of exercise on spontaneous calorie intake in obesity. Am J Clin Nutr 1982;36:470-477.

28 McAuley KA, Smith KJ, Taylor RW, McLay RT, Williams SM, Mann JI: Long term effects of popular dietary approaches on weight loss and features of insulin resistance. Int J Obes 2006;30:342-349.

-29 Ohkawara K, Tanaka S, Miyachi M, IshikawaTakata K, Tabata I: A dose-response relation between aerobic exercise and visceral fat reduction: systematic review of clinical trials. Int J Obes (Lond) 2007;31:1786-1797.

30 Jakicic J: The role of physical activity in prevention and treatment of body weight gain in adults. J Nutr 2002:132:3826S-3829S
31 Nagashima J, Musha H, Takada H, Takagi K, Mita T, Mochida T, Yoshihisa T, Imagawa $\mathrm{Y}$, Matsumoto N, Ishige N, Fujimaki R, Nakajima H, Murayama M: Three-month exercise and weight loss program improves heart rate recovery in obese persons along with cardiopulmonary function. J Cardiol 2010;56:79-84

32 De Jonge L, Moreira EA, Martin CK, Ravussin E: Impact of 6-month caloric restriction on autonomic nervous system activity in healthy, overweight, individuals. Obesity 2010;18:414-416

33 Poirier P, Hernandez TL, Weil KM, Shepard TJ, Eckel RH: Impact of diet-induced weight loss on the cardiac autonomic nervous system in severe obesity. Obes Res 2003;11:1040-1047.

34 Brinkworth GD, Noakes M, Buckley JD, Clifton PM: Weight loss improves heart rate recovery in overweight and obese men with features of the metabolic syndrome. Am Heart J 2006;152:693.e1-6.

35 Kim MK, Tanaka K, Kim MJ, Matsuo T, Ajisaka $\mathrm{R}$ : Exercise training-induced changes in heart rate recovery in obese men with metabolic syndrome. Metab Syndr Relat Disord 2009;7:469-476.

36 Olive JL, Miller GD: Differential effects of maximal- and moderate-intensity runs on plasma leptin in healthy trained subjects. Nutrition 2001;17:365369.

37 Ben Ali S, Ben Yahia F, Sediri Y, Kallel A, Ftouhi B, Feki M: Gender-specific effect of Pro12 Ala polymorphism in peroxisome proliferator-activated-receptor $\mathrm{y}-2$ gene on obesity risk and leptin levels in Tunisian population. Clin Biochem 2009; 42:1642-1647.

38 Doucet E, St-Pierre S, Almeras N, Mauriege P, Despres J P, Richard D, Bouchard C, Tremblay A: Fasting insulin levels influence plasma leptin levels independently from the contribution of adiposity: evidence from both a cross-sectional and an intervention study. J Clin Endocrinol Metab 2000; 85:4231-4237.

39 de Luis D A, Sagrado M G, Conde R, Aller R, Izaola $\mathrm{O}$ : Changes of ghrelin and leptin in response to hypocaloric diet in obese patients. Nutrition 2008; 24:162-166.
40 Ceddia RB, William WN Jr, Lima FB, Curi R: Leptin inhibits insulin-stimulated incorporation of glucose into lipids and stimulates glucose decarboxylation in isolated rat adipocytes. J Endocrinol 1998;158:R7-9.

41 Steinberg GR, Bonen A, Dyck DJ: Fatty acid oxidation and triacylglycerol hydrolysis are inhanced after chronic leptin treatment in rats. Am J Physiol Endocrinol Metab 2002;282:E593-600.

42 Jéquier E: Leptin signaling, adiposity, and energy balance Ann N Y Sci 2002;967:379-388

43 Straznicky NE, Lambert EA, Lambert GW, Masuo K, Esler MD and Nestel PJ: Effects of dietary weight loss on sympathetic activity and cardiac risk factors associated with the metabolic syndrome. J Clin Endocrin Metabol 2005;90:5998-6005.

44 Richards RJ, Blalock A, Liao J and Reisin E: Leptin: Sympathetic and cardiovascular effects. Curr Cardio Rep 2003;56:453-458.

45 Ben Ounis O, Elloumi M, Ben Chiekh I, Zbidi A, Amri M, Lac G, Tabka Z: Effects of two-month physical-endurance and diet-restriction programmes on lipid profiles and insulin resistance in obese adolescent boys. Diabetes Metab 2008;34(6 Pt 1): 595-600.

46 Bond Brill J, Perry AC, Parker L, Robinson A Burnett K: Dose-response effect of walking exercise on weight loss. How much is enough? Int $\mathrm{J}$ Obes Relat Metab Disord: 2002;26:1484-1493.

47 Tzotzas T, Filippatos TD, Triantos A, Bruckert E, Tselepis AD, Kiortsis DN: Effects of a low-calorie diet associated with weight loss on lipoproteinassociated phospholipase A2 (Lp-PLA2) activity in healthy obese women. Nutr Metab Cardiovasc Dis 2008;18:477-482.

48 Zarins ZA, Wallis GA, Faghihnia N, Johnson ML, Fattor JA, Horning MA, Brooks GA: Effects of endurance training on cardiorespiratory fitness and substrate partitioning in postmenopausal women. Metabolism 2009;58:1338-1346.

49 Usherwood JR, Bertram JE: Gait transition cost in humans. Eur J Appl Physiol 2003;90:647-650.

50 Kyröläinen H, Belli A, Komi PV: Biomechanical factors affecting running economy. Med Sci Sports Exerc 2001;33:1330-1337. 